\title{
How many giraffes are there? A comparison . of abundance estimators at Ongava Game Reserve, Namibia
}

\author{
Bonenfant Christophe ${ }^{1}$, Stratford $\mathrm{Ken}^{2}$, and Périquet \\ STÉPHANIE 2
}

${ }^{1}$ (Corresponding author, orcid . org/0000-0002-9924-419X) Université de Lyon, F-69 000, Lyon ; Université Lyon 1 ; CNRS, UMR 5558, Laboratoire de Biométrie et 4 Biologie Évolutive, F-69 622, Villeurbanne, France. E-mail:

christophe.bonenfant@univ-lyon 1.fr

${ }^{2}$ Ongava Research Centre, Private Bag 12041, Ausspannplatz, Windhoek 9000, Namibia.

${ }^{2}$ Ongava Research Centre, Private Bag 12041, Ausspannplatz, Windhoek 9000, Namibia.

5181 (errors:5) words 
Abstract Camera-traps are a versatile and widely adopted tool to collect

2 biological data in wildlife conservation and management. If estimating population abundance from camera-trap data is the primarily goal of many

4 projects, what population estimator is suitable for such data needs to be investigated. We took advantage of a 21 days camera-trap monitoring on giraffes

6 at Onvaga Game Reserve, Namibia to compare capture-recapture (CR), saturation curves and $N$-mixture estimators of population abundance. A marked

8 variation in detection probability of giraffes was observed in time and between individuals. Giraffes were also less likely to be detected after they were seen at a waterhole with cameras (visit frequency of $f=0.25$ ). We estimated population size to 119 giraffes with a $C_{v}=0.10$ with the best CR estimator. All other estimators we a applied over-estimated population size by $c a$. 20 to $>80 \%$, because they did not account for the main sources of heterogeneity in detection probability. We found that modelling choices was much less forgiving for $N$-mixture than CR estimators. Double counts were problematic for $N$-mixture models, challenging the use of raw counts at waterholes to monitor giraffes abundance. 


\section{Introduction}

2

4

The on-going development and large-scale deployment of camera trapping technology offers a promising and appealing way for ecologists to collect a variety of biological data at an unprecedented scale and speed (Swanson et al. 2015). Habitat use, activity patterns and population abundance are now frequently recorded from camera trap data (O'Connell et al. 2011; Trolliet et al. 2014). Sampling a population with camera-traps is indeed particularly useful and efficient (), and even more so for species with idiosyncratic coat or fur patterns from which individual identification is possible (e.g. Jackson et al. 2006, for an application of the elusive snow leopard Panthera uncia). More and more, camera trap data are used to estimate population abundance (Burton et al. 2015; Gilbert et al. 2021) but such data come with specific problems too. Detection rate is not perfect and, sampling design and effort are likely different from physical captures (Hamel et al. 2013; Gilbert et al. 2021). If obtaining unbiased estimates of abundance is of central importance for conservation and wildlife management to set appropriate goals and policies (Anderson 2001), what currently available population abundance estimator is most suitable for camera-trap data remains to be evaluated empirically.

Since long, the underestimation of population abundance has been reported in the wild (Strandgaard 1967; Apollonio et al. 2010). This phenomenon arises because an unknown proportion of animals are missed during surveys, i.e. animal detection is not perfect. Imperfect detection is the main reason why detection probability of individuals underpins most population abundance estimators (Seber 1982; Schwarz \& Seber 1999). Past empirical studies evidenced how variable in time and space detection probability could be in the wild (ref). For instance, detection probability of animals was reported to increase with habitat openness (Choquenot 1995), to decrease with the distance of animals from the observer (Burnham et al. 1980; Buckland et al. 2000) and, between observers themselves depending on their experience or motivation in spotting animals (Collier et al. 2007), or between animals with different personnalities (ref).

Accounting for this intrinsinc and extrinsic sources of detection heterogeneity of animals has profound consequences on the accuracy and precision of population abundance (Veech et al. 2016). Currently, only a handful of population abundance estimators can account for the several sources of variability of detection probability, and most derive from distance sampling (DS) and capture-recapture (CR). Both families of estimators can accommodate 
detection rate for known sources of variability like time of the year, habitat type, or sex and age of individuals (Pollock 1980; Schwarz \& Seber 1999), but only the CR approach can model unmeasured or unknown sources of heterogeneity. That these two methodologies are not systematically implemented in the field comes from serious practical limitations. CR requires a substantial proportion of the population to be recognizable (Strandgaard (1972) advised up to $2 / 3$ of a roe deer population to be marked) achieved by the physical capture of animals to mark them (physically or visually). In addition the capture and marking of wild animals can raise ethical questions for endangered species. DS on the other hand is quite sensitive to the sampling designs (linear transects), which is sometime difficult to carry out in the dense tropical forests of Africa (Duckworth 1998), and the behavioural response of animals to human disturbance (see Elenga et al. 2020 , on duikers). In other words these reference methods to estimate animal abundance can rapidly become prohibitively expensive, time consuming and difficult to implement for wildlife managers (Morellet et al. 2007).

By seeking to keep implementation costs low, practitioners often make use of easier-to-implement, cheaper methods to monitor wildlife populations at spatial scales compatible with wildlife management (Morellet et al. 2007). This legitimate choice often comes at the costs of using less flexible estimators of population abundance to account for variability in detection rate. For instance, the catch-per-unit effort (Leslie \& Davis 1939) or the saturation curves (Petit \& Valière 2006) can return an estimate of population size from unmarked animals but assume constant detection rates for all individuals and over the sampling period. A noticeable exception is $N$-mixture model (Royle 2004), which allows to separate population size from detection probability from repeated counts of animals in time and space. The robustness and accuracy of $N$-mixture abundance estimators is, however, frequently questioned (Kéry 2018).

For decades in large African national parks, a common practice to monitor wildlife has been to index population abundance of large herbivore species from direct (animals) or indirect observations (tracks, faeces) (Jachmann 2002; 2012). Such index of population size includes road transects counts (visibility issues), aerial counts (visibility issues and costs), waterhole counts of various duration (risk of missing water independent species). The underlying assumption of a constant detection rate has been advanced to be the main reason for indices of population abundance failing to track wildlife abundance reliably (Anderson 2001), and its recommendation as management tool is sometimes proposed after a validation test against a reference method (Morellet et al. 2007). If several studies did show that not accounting for detection variability could indeed bias 
population abundance estimates (Dail \& Madsen 2011), the magnitude and direction is this bias is seldom quantified empirically.

Giraffe (Giraffa camelopardalis) is a charismatic species of conservation significance with decreasing populations in many parts of Africa (O'connor et al. 2019). The assessment of local conservation status of populations and their long-term viability are however hampered by the many different ways abundance has been estimated between study areas. Here, we propose to take advantage of

\section{Study area}

the waterhole monitoring with camera traps on Ongava Game Reserve, Namibia, to compare six population size estimators of giraffe population to characterize the biases associated with spatial, temporal and individual variability in detection rates. Being water dependent but with a capacity to spend several days without drinking, individual giraffes come to drink every two or three days (Shorrocks 2016). This behaviour can potentially generate variation in detection probability once individuals visited a waterhole, i.e. an individual seen on a given day will be less likely to be seen on the following day. It is known that males and females have different behaviours and resource requirements (see Gaillard et al. 2003, for examples in different large herbivore species), therefore the frequency of waterhole visit might differ between sexes Shorrocks (2016).

A practical advantage of giraffe is that one can use its idiosyncratic coat patterns to uniquely identify individuals from photographs and then apply $\mathrm{CR}$ estimator to estimate population abundance (Brown et al. 2019). This offers a unique opportunity to quantify the impact of detection heterogeneity on population size estimates, and to assess the relevance of simpler indices of abundance to monitor giraffe populations. We compared the abundance estimates we obtain from CR methodologies, which we regard as the reference method, with $N$-mixture estimates, saturation curves, and raw count data (by observers) on Ongava Game Reserve in year 2016.

\section{Material and Methods}

Ongava Game Reserve (OGR) is located in Namibia, immediately to the south of Etosha National Park (ENP) with a common boundary on Ongava's north side (Fig. 1), covering an area of approximately $300 \mathrm{~km}^{2}$. OGR is surrounded by electrified fenced preventing movement of ungulates in and out the protected area. The habitat is termed Karstveld, with vegetation primarily Colophospermum mopane shrub and woodland, with some areas savannah-like. 
OGR relief is mostly dolomites hills, with a few small open plain areas and a well-defined ridge and small mountain in the central and northern part of the reserve. The weather zone for the reserve is typical for semi-arid northern

4 Namibia, with an average annual rainfall of 380mm (see Stratford \& Stratford 2011, for further details)). There are several natural dams on the reserve, the March). During the dry season (May to December) water is only available at 12 8 artificial waterholes. Ongava supports a range of mammalian herbivores including white and black rhinos, plain zebras, impalas, springboks (Stratford \& Stratford 2011).

\section{Count data}

12 From the $6^{\text {th }}$ to the $27^{\text {th }}$ of September (a total of 22 days) 2016, between three and eight camera traps (Reconyx RC-55 and HC-500 and Bushnell Trophy series) were deployed at each waterhole to monitor their usage by wildlife (see Table S1). Each camera was mounted inside a stainless-steel protection case were set to record a sequence of 10 images with a delay of 30 seconds between sequences, while Bushnell cameras recorded sequences of 3 images with a delay of 15 seconds. We extracted images containing giraffes and their associated metadata (date and time).

Pictures were not taken by human observers, so we only identified

\section{Population size estimations}

\section{Capture-Recapture models}

From giraffe identification on photographs, we built capture histories of $n=101$ individuals over the $t=22$ days of the camera trap survey. We then analysed 
giraffe capture histories with CR methods (Lebreton et al. 1992) in a Bayesian

2 framework (see Kéry \& Schaub 2011). Each giraffe observation at a waterhole is the product of survival $(\phi)$ and detection $(p)$ probabilities, conditional on first

4 observation. We modelled detection probability $p$ on the logit scale as a function of time (categorical variable with 19 levels), whether the giraffe has been seen at

6 any waterhole the previous day or not (categorical variable with 2 levels), and of the total number of functioning cameras (covariate). We also included random

8 effects of the individual $\left(\sigma_{i d}^{2}\right)$ and of time $\left(\sigma_{t}^{2}\right)$. Because we could not sex 8 individuals from photographs, we treated the giraffe sex as a latent Bernouilli variable $S_{i}$ of parameter $\pi$ corresponding to the population sex-ratio. We then entered $S_{i}$ as an explanatory variable (categorical variable with 2 levels) of $p$.

Taken together, our set of fitted models covered the standard estimators for population size namely $\mathrm{M}_{t}$ (time effect), $\mathrm{M}_{t h}$ (time and individual heterogeneity effects) and $\mathbf{M}_{t b h}$ (time, individual heterogeneity and behavioural effects: see Otis et al. 1978). We selected the statistically significant variables from the posterior parameter distributions and only kept variables for which 0 was outside of the $95 \%$ credible interval. In our case, survival rate $\phi$ was of no biological interest and kept constant in time and across individuals. We derived population sizes at each time step $\left(N_{t}\right)$ by dividing the number of detected giraffes at time $t$ by the average detection probability $p$ at time $t+1$ (Burnham et al. 1987).

We considered the $21 N_{t}$ values as the realisation of an unobserved random variable of mean $\left(N_{c}\right)$, the estimated population size of giraffes, and of variance $\sigma_{N}^{2}$, known as a Kalman filter (Kalman 1960; see Wang 2007 for an application in population ecology). We used an open population model because, despite Ongava is a thoroughly fenced reserve, we could not completely ensure that all animal were at risk of observation for the 20 days of survey and giraffes getting in and out of the reserve could not completely be ruled out.

\section{Saturation curves}

We also estimated population size using the saturation curves method (see Petit different giraffes seen at the waterholes (hereafter noted $C_{t}$ ) increased from day 1 to day 20 (see Fig. 2). The addition of new individuals however decreases regularly with time and is expected to level off because few new individuals will be discovered when approaching population size. We can use this curvature to fit a function with an asymptotic number of different individuals and get an estimate of giraffe population size $\left(N_{s}\right)$. Saturation curves have been used 
similarly for decades to estimate species diversity (Colwell \& Coddington 1994).

2 So far two different non-linear functions have been proposed in the literature for the case of population size estimation:

1. the hyperbolic function (Kohn et al. 1999): $C_{t}=\left(N_{s} \times t\right) /(b-t)$;

2. the exponential function (Eggert et al. 2003): $C_{t}=N_{s} \times\left(1-e^{-c \times t}\right)$;

where $t$ is time in days ranging from 1 to 20, and $b$ and $c$ are a breakage parameters, i.e. the rate of decrease of the number of new individuals adding up

A third population size estimator we could apply to our giraffe sampling and data giraffes $C_{t}$ in a Bayesian model to produce another estimate of giraffe population size at Ongava. Note that this approach assumes a constant detection rate over time, space and between individuals, given by $p_{s}=C_{20} / N_{s}$.

\section{N-mixture models}

was the $N$-mixture model (Royle 2004). The $N$-mixture model assumes that repeated counts of animals in time and space are the outcome of combined probability models for the unknown population abundance $\left(N_{N}\right)$ and for the detection $\left(p_{N}\right)$. For population abundance, the Poisson, negative binomial and zero-inflated Poisson distributions are the most commonly used, but other discrete distributions may be considered (see below). For the detection process, a binomial distribution (with parameters $N_{N}$ and $p_{N}$ ) accounts for undetected animals. The $N$-mixture model assumes a demographically closed population and an equal detection probability for all individuals. We estimated giraffe population size by fitting four $N$-mixture models to the giraffe data ( $t=20$ days, $s=12$ waterholes), allowing for temporal variation in detection probabilities (Kéry et al. 2009).

We replicated the analyses of population size estimation for two data sets. The first data set consisted in the number of different and uniquely recognized giraffes seen per day at each of the 12 waterholes. For this data set, we used a binomial distribution to model the observation process. For this data set, we considered another distribution mixture accounting for the non-independence between individuals, the $\beta$-binomial-binomial $N$-mixture models (Martin et al. 2011). We discarded the zero inflated Poisson - binomial mixture because of its poor performance in general (Veech et al. 2016). For the second data set, we used the total number of giraffes seen (without individual recognition) and was 
hence more closely related to counts carried out in many reserves where individuals identification is not done. Here, we used a Poisson model for the observation process because double counts were very frequent from camera-trap photographs ending up with a Poisson-Poisson distribution mixture (Kéry \& Royle 2020). To achieve convergence and facilitate parameter estimations, we included a temporal correlation for detection rates (first order autoregressive model, see Kéry \& Royle 2020, p. XXX). Note that in the case of Poisson -

8 Poisson $N$-mixture models, we do no longer estimate a detection probability $(0<p<1)$ but a detection rate instead $(\psi>0)$.

We fitted all models using JAGS 4.0 (Plummer et al. 2003). We used non-informative prior distributions for all estimated parameters except for $N_{s}$ in

\section{Camera trap data set}

Giraffes were recorded at 10 of the 12 waterholes surveyed. A total of 101 individuals were identified from the camera trap images: 58 adult females, 41 adult males and two juvenile of unknown sex. For all but six individuals we obtained identification from both sides of the animal. For five individuals, we only had picture from the left side and only a front shot for the remaining animal. The majority of individuals $(66 \%, n=58)$ were seen at a single waterhole, while $27 \%(n=24)$ and $7 \%(n=6)$ were seen at two and three waterholes respectively. On average, 28.15 unique giraffes have been detected at OGR per day, with a minimum of 8 and a maximum of 54 (median of 29.5 individuals).

\section{Population size estimates}

From capture histories of individually identified giraffes, the best model describing the observed variability in detection rate of giraffes at Ongava 
included time variation (i.e. differences in detection probability between days), sex and whether a giraffe was seen at any waterholes the day before. We detected a marked variability in daily detection probabilities over the course the of the 4 study, ranging from $\hat{p}={ }_{0.00} 0.04_{0.15}$ on day 2 , to $\hat{p}={ }_{0.27} 0.35_{0.44}$ on day 15 . A female giraffe was 1.46 times more likely to be detected at any waterhole than a male giraffe (sex effect: $\hat{\beta}={ }_{0.02} 0.38{ }_{0.75}$ ). In addition, a giraffe was 0.63 times less likely to be detected in the reserve if it was seen the day before $\left(\hat{\beta}={ }_{-0.92}-0.45-0.01\right)$. Once time, sex and previous visit have been accounted for, the remaining individual heterogeneity in detection rate was $\left(\hat{\sigma}_{i d}^{2}=0.140 .35{ }_{0.67}\right)$. The population size estimate returned from our best model of detection rate was $\hat{N}={ }_{102} 119{ }_{146}$ individuals on OGR (Table 1).

We then calculated the cumulative number of newly detected giraffes over the 20 days duration of the study (Fig.2). The number of new giraffes increased steeply up from day 1 to day 16 when it started to level off. It took 19 days to observe all the individuals identified during the study period (Fig.2). At the end of monitoring, 101 uniquely identified animals were detected. Fitting the hyperpolic and exponential saturation curves to estimate giraffe population size on OGR gave contrasting results (Table 1). While the exponential equation returned a population size of $104117{ }_{134}$ giraffes, the hyperbolic equation projected a population size $49 \%$ larger $\left({ }_{145} 175_{215}\right)$. Breakage coefficients read $\hat{b}={ }_{0.09} 0.12_{0.16}$ and $\hat{c}={ }_{7.9} 11.917 .7$ for the exponential and hyperbolic equations respectively. Overall, the goodness-of-fit of the two saturation curves to the data was poor with $\chi_{d f=21}^{2}=339.4$ and $\chi_{d f=21}^{2}=330.1$ for the exponential and hyperbolic equations (Fig. 2). Precision of the estimates was of the same magnitude, close to $10 \%$ for both models (Table 1).

Finally we applied three different $N$-mixture models to the giraffe data to estimate giraffe population size yielding contrasting results. From the $\hat{N}_{P B}={ }_{173} 215{ }_{263}$ (Table 1), hence $80 \%$ larger than our best CMR estimates. The $\beta$-binomial - binomial mixture estimated abundance to $\hat{N}_{\beta B B}={ }_{107} 124156$ giraffes. The associated parameters of the $\beta$-binomial function were $\hat{\alpha}={ }_{0.32} 0.44_{0.58}$ and $\hat{\beta}={ }_{1.13} 1.82_{2.91}$, giving a correlation $\hat{\rho}={ }_{0.22} 0.31_{0.40}$. According to this model, the daily detection probability was $\hat{p}=0.140 .20_{0.25}$, ranging between $\hat{p}={ }_{0.14} 0.20_{0.25}$ and $\hat{p}={ }_{0.14} 0.20_{0.25}$. Fitting a Poisson-Poisson $N$-mixture model to raw observations led to an estimated population size of $\hat{N}_{P P}={ }_{79} 8799$ giraffes (Table 1). The mean detection rate was $\hat{\psi}=0.55$ but varied from $\hat{\psi_{k, t}}={ }_{0.00} 0.01_{0.03}$ to $\hat{\psi_{k, t}}={ }_{1.79} 3.11_{5.10}$ according to time and space. The first order temporal auto-correlation coefficient (AR(1)) was estimated to 
$\hat{\tau}={ }_{-1.00}-0.56-0.03$. Note that the Poisson-Poisson model was particularly

difficult to fit to our data as we experienced many convergence issues.

\section{Frequency of waterhole visits}

4 We computed the mean time of return to a waterhole and frequency of visits from the daily probabilities as estimated from the CR model. To do so, we

6 simulated 5000 capture histories from a multinomial distribution which parameters are the observed detection probabilities at each day. For each capture history we calculated the difference in days between successive visits, and its inverse to get the frequency of visits. The mean time of return to a waterhole of giraffes at Ongava was $T_{l}={ }_{1.60} 5.00_{14.0}$ days for males, yielding a frequency of $f={ }_{0.07} 0.26{ }_{0.62}$. Females tended to visit waterholes more frequently with a mean time lag of $T_{l}={ }_{1.70} 4.00{ }_{9.5}$ days between two observations, and a frequency $f={ }_{0.10} 0.300_{0.58}$ over 22 days of monitoring.

\section{Discussion}

Population abundance is the core state variable of population dynamics from

which the population growth rates is usually derived (Caughley 1977). Our study system at OGR offers a unique opportunity to apply and compare different methods to estimating giraffe abundance. Being fenced, usual methodological issues arising in demographically open populations are controlled for. Because giraffes are recognizable from their coat patterns, we could apply methods based on the re-observations of individuals (capture-recapture sensu largo) to be compared with other abundance estimators traditionnaly used in wildlife monitoring in African national parks (Jachmann 2012). Taking the CR abundance estimator as the most robust and reference value, simple counts of giraffes at waterholes underestimate the number of giraffes at OGR by ca. $-20 \%$ as we could expect when detection of animals is imperfect (Seber 1982; MacKenzie et al. 2005). In comparison, the other abundance estimators we applied on the same data deviated from -27 to $+80 \%$ from the CR estimate. We warn against substantial risk of over-estimating giraffe abundance when using $\mathrm{N}$-mixture models or saturation curves at large scale and for conservation purposes. By generating marked heterogeneity in detection probability in time and among individuals, the drinking behaviour of giraffes likely accounts for the discrepancies we report among abundance estimators, and should be carefully considered for other species monitored at waterholes. 
Logistic constrains, local habits and the plethora of available methods to

2

4

6

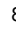
estimate population abundance (Seber 1982) has led to inconsistent ways of monitoring wild populations of large herbivores among and, sometimes, within sites. For instance, at Hwange National Park, Zimbabwe, giraffe density was derived from distance sampling (Valeix et al. 2008), while in the Serengeti aerial counts were preferred as an index of abundance (Strauss 2014, see also Table 1 for an overview). We show here that the choice of a particular method to estimate giraffe abundance has profound consequences on the results. At OGR, the range of estimated population sizes varied more than twofold from 87 to 215 giraffes, yielding densities of 0.29 and 0.71 individuals. $\mathrm{km}^{-2}$. What estimator to implement and to apply to empirical data is all but trivial and comparisons of results with well-known, reference methods is to be advised (e.g. Corlatti et al. 2017; Pellerin et al. 2017). In our case and in the absence of knowledge about the true number of giraffes at OGR, we considered the population size of 119 giraffes (density of 0.39 individuals. $\mathrm{km}^{-2}$ ) derived from CR models to be the most reliable among all estimates. CR methods usually are regarded as the gold standard because of their flexibility in dealing with detection probability and the long history of use since the publication of its principle by Petersen-Lincoln.

If population size as estimated from Eggert's equation is close to CR models (117 vs. 119), the estimation from Kohn's equation seems biologically unrealistic and should be disregarded (see also Frantz \& Roper 2006, for similar results on simulated data). With 175 individuals, giraffe density at OGR $(0.58$ individuals. $\mathrm{km}^{-2}$ ) would hit the upper range of previously reported values for giraffe density across Africa (Table 2). Such a high population density should trigger density-dependent processes, first manifested by a reduction in reproduction rates of females or low juvenile survival in large herbivores (Bonenfant et al. 2009). Still observations of females with a calf at heel at OGR are regularly reported (KS pers. obs.), including in the present data. Saturation curves were shown to give biased estimation of biodiversity when species are not uniformly distributed in space (Collins \& Simberloff 2009). Similarly, projecting the number of total individuals from rarefaction curves (e.g. Petit \& Valière 2006) is likely influenced by heterogeneity in detection probability among individuals. While Kohn's equation returned too a large number of giraffes compared to the CR estimate, Eggert's equation almost matched our reference population size. However, with no replication of our observations and counts, we could not conclude on the robustness of Eggert's equation to heterogeneity in detection probability among individuals. All in all, the fit of the two saturation curves to our giraffe data are poor (Fig. 2) making the inference on population 
size spurious at best.

2 (ref), its reliability is regularly questioned (Dennis et al. 2015; Link et al. 2018;

4

Although $N$-mixture models are more and more used to analyse count data Knape et al. 2018; Nakashima 2020). Comparisons with other proven methods such as CR are scares despite it might help at testing the usefulness of $N$-mixture models for different species. For giraffes, the estimation of population abundance from $N$-mixture models suffers from a severe overestimation (215 for the Poisson-binomial) to an underestimation (87 for the Poisson-Poisson) when applied on raw, unprocessed data without identification of giraffes. If this preliminary processing of data is not possible, double counts likely occurs and a Poisson-Poisson $N$-mixture should work by estimating a detection rate $(\psi>1)$ instead of a detection probability. Double counts is likely the most commonly encountered situation in count operation at waterholes in many African parks. Unfortunately, our results at OGR suggests Poisson-Poisson $N$-mixture model is not to be advised to estimate giraffe population size by returning a number $<$ than the number of different individuals seen (101 giraffes). Despite the occurrence of frequent double counts (empirical rate: 568/101 $=5.62$ from CR data), the Poisson-Poisson $N$-mixture model failed to quantify this quantity correctly $(\hat{\psi}=0.55)$, maybe because of unmodelled heterogeneity, in addition to temporal and spatial variation in the detection probability of animals. Giraffes living in groups, we face non-independence of individual detection which, when accounted for with a $\beta$-binomial distribution in the $N$-mixture model (Martin et al. 2011), returns much more sensible estimates of population size (124 individuals) than any other assumed distributions of animal numbers (Table 1).

A strength of the CR estimator of population abundance over the saturation curves and $\mathrm{N}$-mixture models are their ability to model detection probabilities not only in time and space, but also at the individual level. An important source of heterogeneity in detection probability we observed is the frequency of visit to waterholes by giraffes. Giraffes visit to waterholes is primarily motivated by thirst, and if they must drink on a regular basis they can skip drinking for several days in a row (Shorrocks 2016). At OGR, giraffe return frequency to waterholes was between 0.26 and 0.30 for males and females (one visit every 4-5 days on average), which is lower than previously observed. For instance Shorrocks (2016) reported a frequency of 0.61, while (Caister et al. 2003) recorded daily drinking in Niger $(f \approx 1)$. Such a marked difference in drinking frequency may have biological and technical explanations. Giraffes at OGR may find enough water in forage or access to small, non-monitored waters, making the need to visit larger but dangerous waterholes less stringent. An alternative would be that 
camera traps might fail to trigger in the presence of an animal which is sensitive to camera placement, settings and performance (Rovero et al. 2013; McIntyre et al. 2020), or because the photograph can be of a too low quality to allow for individual identification (e.g. blurry or too dark images). Independently of its cause, this behaviour generates a particular detection pattern as once an individual came to drink, it will be less likely to be detected the following days therefore breaking the assumption of constant detectability of CR models. In the CR terminology, giraffes are "trap shy" and several solutions have been proposed by statisticians to reduce bias on abundance estimates (Pollock 1980).

Our study at OGR is a clear illustration that the assumption of a constant detection rate is not met, even at the relatively short scale of a week. At OGR, detection probability of giraffes varied substantially from one day to another, ranging from 0.04 to 0.35 . This result is a major warning against the use of single, raw count data to monitor giraffes population in the wild such as the number of seen giraffes per day (see Anderson 2001, for a general argument). Variation in daily detection probability resulted from the drinking and grouping behaviour of giraffes, but also from the number of camera traps in service over the course of the study. Several cameras stopped shooting pictures because of battery failure or full memory cards. A sampling design based on fixed camera traps at waterholes hence does not guaranty a constant detectability of giraffes. This marked variability in detection probability in time likely accounts for the discrepancy we report among the five population abundance estimators we applied. In practice, estimating abundance of giraffe populations should preferably consider methods flexible enough to account for the drinking behaviour of giraffes. Clearly, this may apply to other species such as zebras, kudus, wildebeest or bushbuck that all bear idiosyncratic marks.

Sampling large mammal populations with camera traps is of great practical advantage. When it comes to estimation of population abundance from camera-trap data, the long-standing issues of detection and the modelling of its heterogeneity in time, space and among individuals still apply. We found the deviation of $N$-mixture and rarefaction curve models from our reference CR estimation worsen as data are not processed of individual identification. With the automating of animal re-identification from coat and fur patterns with computer vision (Miele et al. 2021), this formerly time consuming processing of images data is now performed in a couple of hours. We believe the gain in precision is worth the time allocated to it.

Acknowledgements We are grateful to Jean-Michel Gaillard for commenting 
on a previous draft of the ms.

2 Author contributions Analysis, writing: CB, SP; study design: KS; field work: SP, KS; revision, editing: CB, SP, KS.

4 Conflicts of interest None.

\section{Ethical standards Of course.}

6

Anderson, D.R. (2001) The need to get the basics right in wildlife field studies. Widlife

8

Apollonio, M., Putman, R., Grignolio, S., Bartoš, L. et al. (2010) Hunting seasons in

10

relation to biological breeding seasons and the implications for the control or regulation of ungulate populations. pp. 80-105. Cambridge University Press.

Bonenfant, c., Gaillard, J.M., Coulson, T., Festa-Bianchet, M., Loison, A., Garel, M. et al. (2009) Empirical evidence of density-dependence in popualtions of large herbivores. Adv. Ecol. Res., 41, 313-357.

Brand, R. (2007) Evolutionary ecology of giraffes (Giraffa camelopardalis) in Etosha National Park, Namibia. Ph.D. thesis, Newcastle University.

Brown, M.B., Bolger, D.T. \& Fennessy, J. (2019) All the eggs in one basket: a countrywide assessment of current and historical giraffe population distribution in Uganda. Global Ecology and Conservation, 19, e00612.

Buckland, S., Goudie, I. \& Borchers, D. (2000) Wildlife population assessment: past developments and future directions. Biometrics, 65, 1-12.

Burnham, K.P., Anderson, D.R. \& LaAke, J.L. (1980) Estimation of density from line transect sampling of biological populations. Wildlife Monographs, pp. 3-202.

Burnham, K.P., Anderson, D. R. White, G.C., Brownie, C. \& Pollock, K.H. (1987) Design and analysis methods for fish survival experiments based on release-recapture. American Fisheries Society, Bethesda, MD. Kenneth P. Burnham. .. [et al.] American Fisheries Society monograph, 5. Includes index. Bibliography: p. 414-420.

Monograph (American Fisheries Society) ; no. 5. 
Burton, A.C., Neilson, E., Moreira, D., Ladle, A., Steenweg, R., Fisher, J.T. et al. 2 (2015) Wildlife camera trapping: a review and recommendations for linking surveys to ecological processes. Journal of Applied Ecology, 52, 675-685.

4 Caister, L.E., Shields, W.M. \& Gosser, A. (2003) Female tannin avoidance: a possible explanation for habitat and dietary segregation of giraffes (giraffa camelopardalis peralta) in niger. African Journal of Ecology, 41, 201-210.

Caughley, G. (1977) Analysis of vertebrate populations. Wiley, London. Graeme Caughley. 'A Wiley-Interscience Publication' Includes index. Bibliography: p.217-228.

Choquenot, D. (1995) Assessing visibility bias associated with helicopter counts of feral pigs in australia's semi-arid rangelands. Wildlife Research, 22, 569-577.

Collier, B.A., Ditchkoff, S.S., Raglin, J.B. \& Smith, J.M. (2007) Detection probability and sources of variation in white-tailed deer spotlight surveys. The Journal of wildlife management, 71, 277-281.

Collins, M.D. \& Simberloff, D. (2009) Rarefaction and nonrandom spatial dispersion patterns. Environmental and Ecological Statistics, 16, 89-103.

Colwell, R.K. \& Coddington, J.A. (1994) Estimating terrestrial biodiversity through extrapolation. Philosophical Transactions of the Royal Society of London. Series B: Biological Sciences, 345, 101-118.

Corlatti, L., Nelli, L., Bertolini, M., Zibordi, F. \& Pedrotti, L. (2017) A comparison of four different methods to estimate population size of Alpine marmot (Marmota marmota). Hystrix, 28, 61-67.

Crall, J.P., Stewart, C.V., Berger-Wolf, T.Y., Rubenstein, D.I. \& Sundaresan, S.R. (2013) Hotspotter-patterned species instance recognition. In 2013 IEEE workshop on applications of computer vision (WACV), pp. 230-237.

DaIL, D. \& Madsen, L. (2011) Models for estimating abundance from repeated counts of an open metapopulation. Biometrics, 67, 577-587.

Dennis, E.B., Morgan, B.J. \& Ridout, M.S. (2015) Computational aspects of $n$-mixture models. Biometrics, 71, 237-246.

Duckworth, J. (1998) The difficulty of estimating population densities of nocturnal forest mammals from transect counts of animals. Journal of Zoology, 246, 443-486. 
EgGert, L., EgGert, J. \& Woodruff, D. (2003) Estimating population sizes for elusive

2

4 Elenga, G., Bonenfant, C. \& Péron, G. (2020) Distance sampling of duikers in the rainforest: Dealing with transect avoidance. Plos one, 15, e0240049.

6 Frantz, A.C. \& Roper, T.J. (2006) Simulations to assess the performance of different rarefaction methods in estimating population size using small datasets. Conservation

$8 \quad$ Genetics, 7, 315-318.

Gaillard, J.M., Loison, A. \& Tö̈go, C. (2003) Variation in life history traits and realistic population models for wildlife management. pp. 115-132.

Gelman, A. \& Pardoe, I. (2006) Bayesian measures of explained variance and pooling in multilevel (hierarchical) models. Tecnhometrics, 48, 241-252.

Gilbert, N.A., Clare, J.D., Stenglein, J.L. \& Zuckerberg, B. (2021) Abundance estimation of unmarked animals based on camera-trap data. Conservation Biology, 35, $88-100$.

Hamel, S., Killengreen, S.T., Henden, J.A., Eide, N.E., Roed-Eriksen, L., Ims, R.A. et al. (2013) Towards good practice guidance in using camera-traps in ecology: influence of sampling design on validity of ecological inferences. Methods in Ecology and Evolution, 4, 105-113.

20

Hayward, M.W., O’Brien, J. \& Kerley, G.I. (2007) Carrying capacity of large African predators: predictions and tests. Biological Conservation, 139, 219-229.

22

Jachmann, H. (2002) Comparison of aerial counts with ground counts for large african herbivores. Journal of Applied Ecology, 39, 841-852.

JaCHMANN, H. (2012) Estimating abundance of African wildlife: an aid to adaptive management. Springer Science \& Business Media.

Jackson, R.M., Roe, J.D., Wangchuk, R. \& Hunter, D.O. (2006) Estimating snow leopard population abundance using photography and capture-recapture techniques.

28

Kalman, R.E. (1960) A new approach to linear filtering and prediction problems.

30

Kéry, M. \& Schaub, M. (2011) Bayesian population analysis using WinBUGS: a

32

hierarchical perspective. Academic Press. 
Kiffner, C., Binzen, G., Cunningham, L., Jones, M., Spruiell, F. \& Kioko, J. (2020)

2 Wildlife population trends as indicators of protected area effectiveness in northern tanzania. Ecological Indicators, 110, 105903.

4 Knape, J., Arlt, D., Barraquand, F., Berg, Å., Chevalier, M., Pärt, T. et al. (2018) Sensitivity of binomial $n$-mixture models to overdispersion: The importance of assessing model fit. Methods in Ecology and Evolution, 9, 2102-2114.

Kohn, M.H., York, E.C., Kamradt, D.A., Haught, G., Sauvajot, R.M. \& Wayne, R.K. (1999) Estimating population size by genotyping faeces. Proceedings of the Royal Society of London. Series B: Biological Sciences, 266, 657-663.

KéRY, M. (2018) Identifiability in $n$-mixture models: A large-scale screening test with bird data. Ecology, 99, 281-288.

Kéry, M. \& Royle, J.A. (2020) Applied Hierarchical Modeling in Ecology: Analysis of distribution, abundance and species richness in R and BUGS: Volume 2: Dynamic

$14 \quad$ and Advanced Models. Academic Press.

Kéry, M., Royle, J.A., Plattner, M. \& Dorazio, R.M. (2009) Species richness and occupancy estimation in communities subject to temporary emigration. Ecology, 90, 1279-1290.

Lebreton, J.D., Burnham, K.P., Clobert, J. \& Anderson, D.R. (1992) Modeling survival and testing biological hypotheses using marked animals: a unified approach with case studies. Ecological Monographs, 62, 67-118.

LeE, D.E. \& Bond, M.L. (2016) Precision, accuracy, and costs of survey methods for giraffe Giraffa camelopardalis. Journal of Mammalogy, 97, 940-948.

LesLie, P. \& Davis, D. (1939) An attempt to determine the absolute number of rats on a given area. The Journal of Animal Ecology, pp. 94-113.

Link, W.A., Schofield, M.R., Barker, R.J. \& SAUer, J.R. (2018) On the robustness of n-mixture models. Ecology, 99, 1547-1551.

MacKenzie, D.I., Nichols, J.D., Sutton, N., Kawanishi, K. \& Bailey, L.L. (2005) Improving inferences in population studies of rare species that are detected imperfectly. Ecology, 86, 1101-1113.

Martin, J., Royle, J.A., Mackenzie, D.I., Edwards, H.H., Kery, M. \& Gardner, B. (2011) Accounting for non-independent detection when estimating abundance of 32 organisms with a Bayesian approach. Methods in Ecology and Evolution, 2, 595-601. 
McIntyre, T., Majelantle, T., Slip, D. \& Harcourt, R. (2020) Quantifying imperfect

2 camera-trap detection probabilities: implications for density modelling. Wildlife Research, 47, 177-185.

4 Mcqualter, K.N. (2018) The ecology and behaviour of giraffe in Northern Botswana. University of New South Wales, Australia.

6 Miele, V., Dussert, G., Spataro, B., Chamaillé-Jammes, S., Allainé, D. \& Bonenfant, C. (2021) Revisiting animal photo-identification using deep metric learning and network analysis. Methods in Ecology and Evolution, 12, 863-873.

Morellet, N., Gaillard, J.M., Hewison, A.J.M., Ballon, P., Boscardin, Y., Duncan, P. et al. (2007) Indicators of ecological change: new tools for managing populations of large herbivores. J. Appl. Ecol., 44, 634-643.

Mseja, G.A., Kisingo, A.W., Stephan, E. \& Martin, E.H. (2020) Dry season wildlife census in Mkomazi National Park, 2015. In Protected Areas in Northern Tanzania, pp.

$14 \quad 133-143$. Springer.

Muller, Z. (2019) Rothschild's giraffe Giraffa camelopardalis rothschildi (Linnaeus, 1758) in East Africa: A review of population trends, taxonomy and conservation status. African Journal of Ecology, 57, 20-30.

Nakashima, Y. (2020) Potentiality and limitations of $n$-mixture and royle-nichols models to estimate animal abundance based on noninstantaneous point surveys. Population Ecology, 62, 151-157.

Ndiweni, T., Zisadza-Gandiwa, P., Ncube, H., Mashapa, C., Gandiwa, E. et al. (2015) Vigilance behavior and population density of common large herbivores in a southern african savanna. Journal of Animal and Plant Sciences, 25, 876-883.

O’Connell, A., Karanth, K. \& Nichols, J.D., eds. (2011) Camera traps in animal ecology. Springer Japan, Tokyo.

O'connor, D., Stacy-Dawes, J., Muneza, A., Fennessy, J., Gobush, K., Chase, M.J. et al. (2019) Updated geographic range maps for giraffe, Giraffa spp., throughout

28 sub-Saharan Africa, and implications of changing distributions for conservation. Mammal Review, 49, 285-299.

Ogutu, J.O., Owen-Smith, N., Piepho, H.P. \& Said, M.Y. (2011) Continuing wildlife population declines and range contraction in the mara region of kenya during 1977-2009. Journal of Zoology, 285, 99-109. 
Otis, D.L., Burnham, K.P., White, G.C. \& Anderson, D.R. (1978) Statistical inference from capture data on closed animal populations. Wildlife monographs, pp. 3-135.

Pellerin, M., Bessière, A., Maillard, D., Capron, G., Gaillard, J.M., Michallet, J. et al. (2017) Saving time and money by using diurnal vehicle counts to monitor roe deer abundance. Wildlife Biology, 2017.

Petit, E. \& Valière, N. (2006) Estimating population size with noninvasive capture-mark-recapture data. Conserv. Biol., 20, 1062-1073.

Plummer, M. et al. (2003) JAGS: A program for analysis of bayesian graphical models using gibbs sampling. In Proceedings of the 3rd international workshop on distributed statistical computing, vol. 124, pp. 1-10. Vienna, Austria.

Pollock, K.H. (1980) Capture-recapture models: a review of current methods, assumptions and experimental design.

Rovero, F., Zimmermann, F., Berzi, D. \& Meek, P. (2013)" which camera trap type and how many do i need?" a review of camera features and study designs for a range of wildlife research applications. Hystrix, 24.

RoyLe, J.A. (2004) $N$-mixture models for estimating population size from spatially replicated counts. Biometrics, 60, 108-115.

Schwarz, C. \& Seber, G.A.F. (1999) A review of estimating animal abundance III. Statistical Science, 14, 427-456.

Seber, G.A.F. (1982) The estimation of animal abundance and related parameters.

Shorrocks, B. (2016) The giraffe: biology, ecology, evolution and behaviour. John Wiley \& Sons.

StrandgaARd, H. (1967) Reliability of the Petersen method tested on a roe-deer population. Journal of Wildlife Management, 31, 643-651.

StrandgaArd, H. (1972) The roe deer (Capreolus capreolus) population at Kälo and the factors regulating its size. Danish Review of Game Biology, 7, 1-205.

Stratford, K.J. \& Stratford, S.M. (2011) Fine-scale movements and use of space by spotted hyaena (Crocuta crocuta) on Ongava Game Reserve, Namibia. African Journal of Ecology, 49, 343-352.

Strauss, M.K.L. (2014) Ecological and anthropogenic drivers of giraffe (Giraffa camelopardalis tippelskirchi) population dynamics in the Serengeti. Ph.D. thesis, 
Suraud, J.P., Fennessy, J., Bonnaud, E., Issa, A., Fritz, H. \& Gaillard, J.M. (2012)

2 Higher than expected growth rate of the endangered West African giraffe Giraffa camelopardalis peralta: a successful human-wildlife cohabitation. Oryx, 46, 577-583.

4 Swanson, A., Kosmala, M., Lintott, C., Simpson, R., Smith, A. \& Packer, C. (2015) Snapshot Serengeti, high-frequency annotated camera trap images of 40 mammalian $6 \quad$ species in an african savanna. Scientific data, 2, 1-14.

Treydte, A.C., Edwards, P.J. \& Suter, W. (2005) Shifts in native ungulate communities on a former cattle ranch in Tanzania. African Journal of Ecology, 43, 302-311.

Trolliet, F., Vermeulen, C., Huynen, M.C. \& Hambuckers, A. (2014) Use of camera traps for wildlife studies: a review. Biotechnologie, Agronomie, Société et Environnement, 18, 446-454.

Valeix, M., Fritz, H., Chamaillé-Jammes, S., Bourgarel, M. \& Murindagomo, F. (2008) Fluctuations in abundance of large herbivore populations: insights into the influence of dry season rainfall and elephant numbers from long-term data. Animal Conservation, 11, 391-400.

Veech, J.A., Otт, J.R. \& Troy, J.R. (2016) Intrinsic heterogeneity in detection probability and its effect on $\mathrm{N}$-mixture models. Methods in Ecology and Evolution, 7 , 1019-1028.

WANG, G. (2007) On the latent state estimation of nonlinear population dynamics using bayesian and non-bayesian state-space models. Ecological Modelling, 200, 521-528. 
TABLE 1 Estimated population size $(\hat{N})$ of giraffes (Giraffa camelopardalis) at Ongava Game Reserve, Namibia, in September 2016. An intensive monitoring from 10 camera-traps located at waterholes lasted for 21 days. The capture-recapture estimator modelled detection probability of animals accounting for daily variation, sex of individual, and whether the giraffe has previously visited a waterhole the day before or not. For the sake of comparisons, we present the detection probabilities $a$ posteriori as the number of counted animals divided by $\hat{N}$. For $N$-mixture models (re-id), abundance estimation used the number of uniquely identified giraffe each day at every waterhole, hence removing double counts (Poisson-binomial and $\beta$-binomial-binomial mixtures) to return population size and detection probability. Another $N$-mixture model used the raw number of giraffes counted at each waterhole instead (Poisson-Poisson mixture), which is the most common configuration in wildlife counts in Africa. In this case, multiple counts of the same giraffe do occur, which is reflected by the detection rate $>1$ we report (note this is no longer a probability). We report here the point estimate and associated $95 \%$ credible intervals as: lower limit mean upper limit. $C_{v}$ stands for the coefficient of variation of $\hat{N}$.

\begin{tabular}{|c|c|c|c|}
\hline Estimator & $\hat{N}$ & $\bar{p}$ & $C_{v}$ \\
\hline Capture-recapture $p_{t+h}$ & 101111128 & 0.790 .900 .99 & $6.5 \%$ \\
\hline Capture-recapture $p_{t+s e x+h}$ & 101110128 & 0.750 .921 .00 & $7.4 \%$ \\
\hline Capture-recapture $p_{t+s e x+b+h}$ & 102119146 & 0.690 .850 .99 & $6.5 \%$ \\
\hline Saturation curve (Kohn) & 145175215 & 0.470 .570 .70 & $10.0 \%$ \\
\hline Satura & 104117134 & 0.750 .860 .97 & $6.3 \%$ \\
\hline$N$-mix & ${ }_{173} 215263$ & 0.380 .470 .58 & $4.4 \%$ \\
\hline$N$-mixture (ZIP-binomial) & ${ }_{173} 215263$ & 0.380 .470 .58 & $4.4 \%$ \\
\hline$N$-mixture $(\beta$-binomial-binomial $)$ & 107124156 & 0.650 .810 .94 & $10.1 \%$ \\
\hline$N$-mixture (Poisson-Poisson) & 798799 & $1.021 .16_{1.28}$ & $5.4 \%$ \\
\hline
\end{tabular}


TABLE 2 Reported densities of giraffe (Giraffa camelopardalis) populations in Africa (in number of individuals per $\mathrm{km}^{2}$ ). When abundance was estimated for several years, repeated lines in the same location give the range of densities recorded on the site.

\begin{tabular}{|c|c|c|c|}
\hline Site & Country & Density & Reference \\
\hline Tarangire Ecosystem & Tanzania & $0.791(0.073)$ & Lee \& Bond (2016) \\
\hline Tarangire Ecosystem & Tanzania & $1.202(0.760)$ & Lee \& Bond (2016) \\
\hline Tarangire Ecosystem & Tanzania & $0.173(0.057)$ & Lee \& Bond (2016) \\
\hline Serengeti & Tanzania & NA & Strauss 2014 \\
\hline Chobe National Park & Botswana & $0.110(-)$ & Mcqualter (2018) \\
\hline Etosha National Park & Namibia & $0.150(\sqcup)$ & Brand (2007) \\
\hline Etosha National Park & Namibia & $0.200(-)$ & Brand (2007) \\
\hline Gonarezhou National Park & Zimbabwe & $0.470(0.140)$ & Ndiweni et al. (2015) \\
\hline Malipati Sarafi Area & Zimbabwe & $0.010(0.030)$ & Ndiweni et al. (2015) \\
\hline Great Rift Valley & Kenya & $0.468(88 / 188)$ & Muller (2019) \\
\hline Great Rift Valley & Kenya & $0.405(77 / 190)$ & Muller (2019) \\
\hline Mara Region & Kenya & $0.750(\sqcup)$ & Ogutu et al. (2011) \\
\hline Mara Region & Kenya & $0.080(\longrightarrow)$ & Ogutu et al. (2011) \\
\hline Saadani National Park & Tanzania & $0.100-1.400$ & Treydte et al. (2005) \\
\hline Lake Manyara National Park & Tanzania & $0.570(0.570-0.580)$ & Kiffner et al. (2020) \\
\hline Lake Manyara National Park & Tanzania & $1.210(1.180-1.25)$ & Kiffner et al. (2020) \\
\hline Kouré and Fandou Plateaus & Niger & $X X X=193 /$ surface & Suraud et al. (2012) \\
\hline Shamwari & South Africa & $0.744(-)$ & Hayward et al. (2007) \\
\hline Lupande & Zambia & $1.274(930 / 730)$ & Jachmann (2002) \\
\hline Ongava & Namibia & $0.336(-)$ & This study \\
\hline Hwange National Park & Zimbabwe & 0.170 & Valeix et al. (2008) \\
\hline Mkomazi National Park & Tanzania & $1.165(0.808)$ & Mseja et al. (2020) \\
\hline Hluhluwe iMfolozi National Park & South Africa & 0.137 & Hervé Fritz \\
\hline Amboseli National Park & Kenya & 0.133 & Hervé Fritz \\
\hline Katavi National Park & Tanzania & 0.938 & Hervé Fritz \\
\hline Kidepo Valley National Park & Uganda & 0.037 & Hervé Fritz \\
\hline Kruger National Park & South Africa & 0.157 & Hervé Fritz \\
\hline Kruger National Park & South Africa & 0.270 & Hervé Fritz \\
\hline Mkomazi Game Reserve & Tanzania & 0.077 & Hervé Fritz \\
\hline Nairobi National Park & Kenya & 0.623 & Hervé Fritz \\
\hline Okavango Delta & Botswana & 0.250 & Hervé Fritz \\
\hline Pilanesburg National Park & South Africa & 0.286 & Hervé Fritz \\
\hline Savuti area of Chobe National Park & Botswana & 0.720 & Hervé Fritz \\
\hline
\end{tabular}




\section{Figure legend}

2 FIg. 1 Spatial distribution of waterholes surveyed in 2016 with camera traps to monitor widlife abundance at Ongava Game Reserve, Namibia. We used camera

4 trap data to extract abundance data on giraffe (Giraffa camelopardalis) to be apply to different estimators of giraffe population size.

6 FIg. 2 Acquisition curves for new individual giraffes during the 21-days long camera trap survey in 2016 at Ongava reserve, Namibia (step curve in black).

8 Continuous lines and associated shaded areas represent predictions and credible intervals of saturation models. We fitted two saturation equations proposed by

10 Eggert et al. (2003) and Kohn et al. (1999) to the problem of population size estimation using a Bayesian framework. 
bioRxiv preprint doi: https://doi.org/10.1101/2021.09.28.459593; this version posted September 29, 2021. The copyright holder for this preprint (which was not certified by peer review) is the author/funder, who has granted bioRxiv a license to display the preprint in perpetuity. It is made available under aCC-BY-ND 4.0 International license.

Figure 1

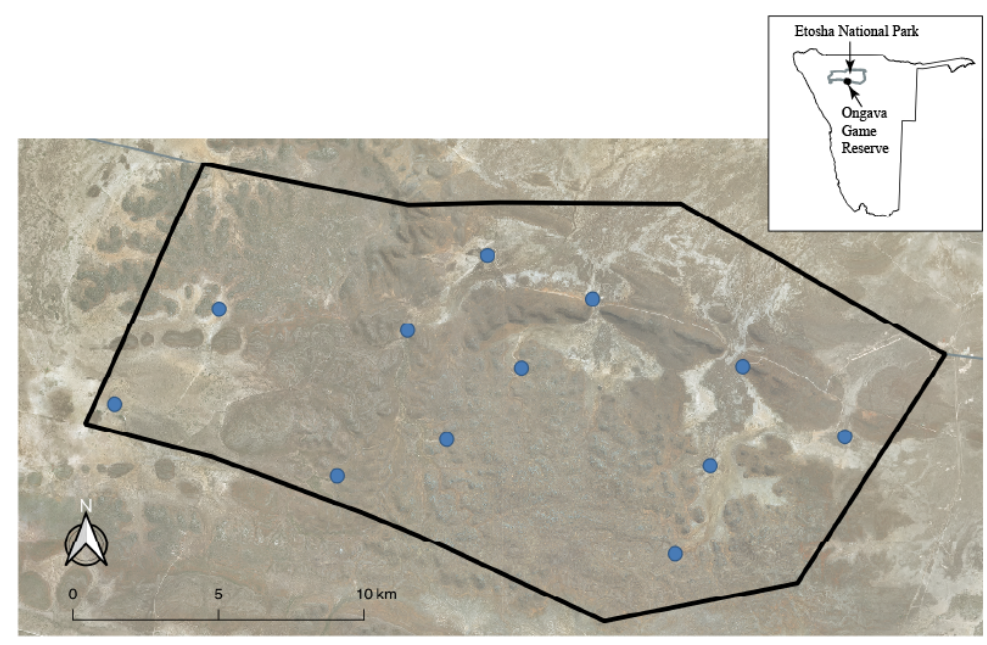


bioRxiv preprint doi: https://doi.org/10.1101/2021.09.28.459593; this version posted September 29, 2021. The copyright holder for this preprint (which was not certified by peer review) is the author/funder, who has granted bioRxiv a license to display the preprint in perpetuity. It is made available under aCC-BY-ND 4.0 International license.

FIGURE 2

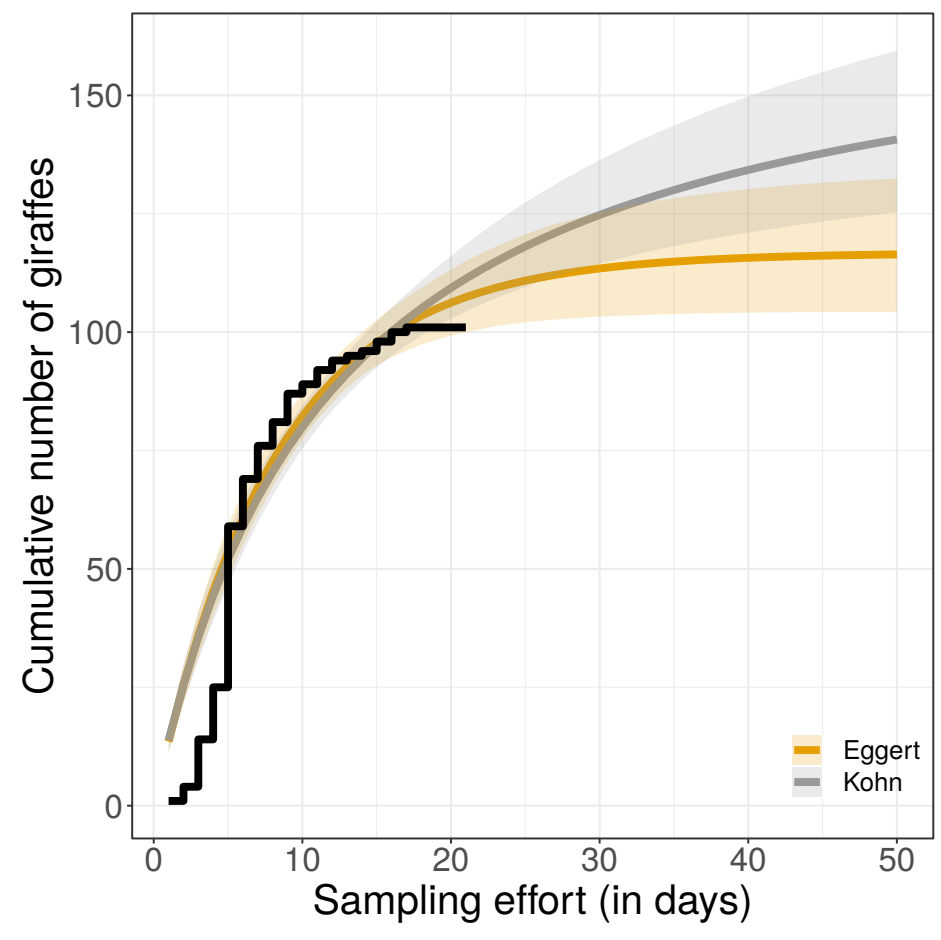

